\title{
Comparison of Blister Aneurysm Treatment Techniques: A Systematic Review and Meta-
}

Analysis

Victoria E. Sanchez BS ${ }^{1}$, Ali S. Haider BS ${ }^{2}$, Scott E. Rowe MS $S^{3}$, Waseem Wahood MS ${ }^{4}$, Navraj S. Sagoo BS $S^{5}$, Ahmad Ozair BS $S^{6}$, Tarek Y. El Ahmadieh $M D^{7}$, Peter Kan $M D^{8}$, Jeremiah N. Johnson $M D^{9}$

${ }^{1}$ Department of Neurosurgery, Indiana University School of Medicine, Indianapolis, IN

${ }^{2}$ Department of Neurosurgery, Texas A\&M University College of Medicine, Bryan, TX

${ }^{3}$ Department of Surgery, Nova Southeastern University College of Osteopathic Medicine, Davie, FL

${ }^{4}$ Department of Surgery, Nova Southeastern University College of Allopathic Medicine, Davie, FL

${ }^{5}$ Department of Orthopaedic Surgery, University of Texas Medical Branch School of Medicine, Galveston, TX

${ }^{6}$ Department of Neurosurgery, King George's Medical University, Lucknow, Uttar Pradesh, India

${ }^{7}$ Department of Neurosurgery, University of Texas Southwestern Medical Center, Dallas, TX

${ }^{8}$ Department of Neurosurgery, University of Texas Medical Branch, Galveston, TX

${ }^{9}$ Department of Neurosurgery, Baylor College of Medicine, Houston, TX

Abstract word count: 244

Text word count: 3455

References: 28

Tables: 2

Figures: 2

Videos: 0

\section{Corresponding Author:}

Jeremiah N. Johnson, MD

Department of Neurosurgery

Baylor College of Medicine

This is the author's manuscript of the article published in final edited form as:

Sanchez, V. E., Haider, A. S., Rowe, S. E., Wahood, W., Sagoo, N. S., Ozair, A., El Ahmadieh, T. Y., Kan, P., \& Johnson, J. N. (2021). Comparison of Blister Aneurysm Treatment Techniques: A Systematic Review and Meta-Analysis. World Neurosurgery. https://doi.org/10.1016/j.wneu.2021.06.129 
7200 Cambridge St., Suite 9B

Houston, TX, USA 77030

Phone: 713-798-6112

Email: jjohnson.neuro@gmail.com

Running Title: Comparison of Blister Aneurysm Treatment Techniques

\section{Disclosure of funding: None}

Acknowledgments: None

Conflict of Interest: The authors have no disclosures related to the content of this manuscript.

Keywords: blood blister aneurysm, flow-diverting stents, endovascular treatment, subarachnoid hemorrhage 


\section{Comparison of Blister Aneurysm Treatment Techniques: A Systematic Review and Meta-}

Analysis

\section{Abstract}

5 Objective: Blood blister aneurysms are small, thin-walled, rapidly growing side-wall aneurysms that have proven particularly difficult to treat, and evidence-based guidance for treatment

7 strategies is lacking. A systematic review and meta-analysis was performed to aggregate the 8 available data and compare the three primary treatment modalities.

9 Methods: We performed a comprehensive literature search according to PRISMA guidelines

10 followed by an indirect meta-analysis that compares the safety and efficacy of surgical, flow-

11 diverting stents (FDS), and other endovascular approaches for the treatment of ruptured blood 12 blister aneurysms.

13 Results: A total of 102 studies were included for quantitative synthesis with sample sizes of 687

14 treated surgically, 704 treated endovascularly without FDS, and 125 treated via flow-diversion.

15 Comparatively, FDS achieved significantly reduced rates of perioperative retreatment compared

16 to both surgical $(P=0.025)$ and non-FDS endovascular $(P<0.001)$. The FDS subgroup also

17 achieved a significantly lower incidence of perioperative rebleed $(P<0.001)$, perioperative

18 hydrocephalus $(P=0.012)$, postoperative infarction $(P=0.002)$, postoperative hydrocephalus

$19(P<0.001)$, and postoperative vasospasm $(P=0.002)$ when compared to those patients in the open

20 surgical subgroup. While no significant differences were found between groups on the basis of

21 functional outcomes, angiographic outcomes detailed by rates of radiographic complete

22 occlusion were highest for surgical $(90.7 \%, 262 / 289)$ and FDS $(89.1 \%, 98 / 110)$ subgroups versus

23 the non-FDS endovascular subgroup at $(82.7 \%, 268 / 324)$. 
24 Conclusion: Flow-diversion appears to be an effective treatment strategy for ruptured BBAs 25 with lower rates of perioperative complications when compared to surgical and other 26 endovascular techniques but studies investigating long-term outcomes following flow-diversion

27 warrant further study.

\section{Introduction}

Blood blister aneurysms (BBAs), as initially described by Takahashi et al., represent a

31 rare but surgically challenging vascular pathology arising from non-branched arteries. ${ }^{1,2}$

32 Although often reported as occurring in the anterior or posterior circulation, most BBAs

33 characteristically present at the supraclinoid segment of the ICA but can occur in other

34 intracranial vessels as well. ${ }^{3}$ They are particularly rare, accounting for approximately $0.3-1 \%$ of

35 all intracranial aneurysms and $6.6 \%$ of ruptured intracranial aneurysms. ${ }^{4,5}$ Patients classically

36 present with an acute subarachnoid hemorrhage (SAH), rapidly evolving clinical course and

37 angiographic imaging demonstrating a small broad-based intracranial arterial wall irregularity.

38 Although pathophysiologically obscure, ruptured BBAs are thought to represent an underlying

39 intracranial arterial dissection and are associated with a high degree of vessel wall fragility and

40 prone to growth and re-rupture. ${ }^{6-9}$

BBAs present a therapeutic challenge because of their difficulty to treat by conventional

42 surgical reconstruction and high susceptibility to intra-operative rupture as well as rebleed

43 postoperatively. ${ }^{4}$ There are many treatment modalities in clinical use for the treatment of BBAs.

44 Patients may be treated surgically by direct clipping, wrapping, trapping with or without bypass,

45 artery occlusion or by endovascular approaches via coils, stent-assisted coiling, multiple

46 overlapping stents or with flow-diverting stents. While various techniques are used to secure 
47 these difficult lesions, no one treatment modality has proven distinctly superior in the literature.

48 Recently, reports of flow-diverting stents used in BBAs have been coupled to lower morbidity

49 and mortality compared to more invasive surgical techniques. ${ }^{10-15}$

50 This systematic review and meta-analysis aims to analyze the growing body of literature

51 reporting outcomes of flow-diverting stents used in the management of BBAs compared to

52 conventional treatment approaches. The clinical indications, outcomes, and potential

53 complications of these procedures are reviewed.

55 Methods

56 Literature Search

57 A systematic review was performed according to the Preferred Reporting Items for

58 Systematic Reviews and Meta-Analyses (PRISMA) guidelines. ${ }^{16}$ A literature search was

59 conducted using the key words "blister aneurysm" entered for search in electronic databases

60 PubMed, Cochrane Central Register of Controlled Trials, and Scopus from dates of their

61 inception to May 2020. In addition, ClinicalTrials.gov was used for access to grey literature. Our

62 electronic search strategy is presented and follows the 2009 PRISMA Group guidelines outlined

63 in Supplemental Figure 1. Identified studies were uploaded into Endnote and duplicates were

64 removed.

65 Study Selection

66 Pre-specified inclusion and exclusion criteria were defined a priori. Studies were eligible

67 for inclusion if they met the following criteria: 1) adult patients (18 or older); 2) English

68 language; 3) available data on ruptured blister aneurysms treated by surgery and/or endovascular;

69 4) adequate data on clinical and/or angiographic outcomes; 5) studies that were retrospective or 
70 prospective with at least one patient. Abstracts, conference presentations, editorials, and animal

71 studies were excluded. Review articles and meta-analyses were also excluded.

72 Two authors (A.S.H. and J.N.J.) independently screened the titles and abstracts of all

73 articles based on the pre-specified inclusion and exclusion criteria. Articles that met the inclusion

74 criteria were then full-text reviewed independently by (V.S., S.R., and A.O.) and eligible articles

75 were selected based on the set criteria. Discrepancies between the authors were resolved by

76 involvement of other authors (A.S.H., J.N.J.). References of relevant articles were also reviewed

77 to ensure no studies were neglected in our electronic search.

\section{Data Extraction}

79 Data were independently extracted by two authors (V.S. and S.R.) using a standardized

80 form, and any disagreement was resolved by consensus. Data were independently confirmed by

81 two other authors (A.S.H and J.N.J). The study design, data extraction, results review, and

82 reporting were overseen by the senior investigator (J.N.J).

83 For each study evaluated, the following data were extracted: study design, patient

84 demographics, comorbidities, presenting clinical status (Hunt and Hess or Fisher grades),

85 aneurysm details (location and size), treatments (clipping and suturing, parent artery occlusion,

86 trapping, wrapping, coil embolization, flow-diverting stent, stent placement alone, stent-assisted

87 coiling, multi-stent overlapping), anti-platelet therapy, complications, outcomes (perioperative

88 mortality or morbidity, early and late angiographic outcomes, clinical outcomes measured by

89 modified Rankin Scale (mRS), postoperative complications, and survival status). Good outcome

90 was defined as an mRS score of 0-2 and poor outcome as mRS score 3-5. For studies in which

91 quantitative measures were not available, favorable neurologic outcome was dependent on terms 
92 such "good recovery". Distinctions between perioperative and postoperative outcomes were

93 defaulted to two weeks after surgery unless otherwise stated by each individual study.

\section{Outcome Measures}

95 Clinical outcomes were extracted for the overall population of patients diagnosed and

96 treated for ruptured blood blister aneurysms. The primary outcome of interest was to perform

97 analyses comparing the clinical outcomes of patients treated with a flow-diverting stents versus

98 surgery and all other endovascular treatments.

99 Statistical Analysis

100 An indirect meta-analysis was performed comparing the outcomes of interest of the three

101 groups. This analyzes each arm separately with their corresponding subgroup pooled result and

102 compares all three results. For any statistically significant comparison, post-hoc analysis was

103 conducted for individual comparisons between the arms. Any study treatment group with a

104 sample size less than 3 was excluded due to limited power of that study. Results were

105 represented by average proportion (effect size, ES) with their corresponding 95\% confidence

106 intervals (CIs). Results were also depicted as forest plots, and heterogeneity between groups

107 indicates statistical significance for all groups' differences. Statistical analysis was performed

108 using STATA 16 (Stata-Corp 2019. STATA Statistical Software: Release 16. College Station,

109 TX: StataCorp LP). P-values $<0.05$ were considered statistically significant.

110 Assessing Risk of Bias of Studies and Results

111 Risk of Bias assessment for quality of evidence of analyzed results was conducted using

112 Grading of Recommendations, Assessment, Development and Evaluations (GRADE), whereby

113 results are assessed based on inconsistency, indirectness, imprecision, and other considerations.

$114{ }^{17}$ Risk of bias assessment for individual studies was conducted using the modified Newcastle- 
115 Ottawa Scale (NOS), whereby “Comparability of Cohorts” was removed in our assessment due 116 to the nature of indirect meta-analysis depicted in Supplemental Tables 1 and 2. ${ }^{18}$

\section{Results}

119 Study selection.

120 The initial literature search identified 762 studies using PubMed, Scopus, Cochrane, and 121 Clinicaltrials.gov (Supplemental Figure 1). After duplicates were removed, 440 articles were left

122 for review. An additional 61 records were removed at this stage via thorough inspection reaching 123 a total of 379 results. Overall, 191 studies met inclusion and exclusion criteria, were full-text

124 reviewed, and data were compiled for qualitative synthesis. For meta-analysis, pre-specified 125 eligibility required at least 3 patients per treatment group with adequate reports of angiographic 126 outcome, leaving a total of 102 studies for quantitative synthesis.

127 Patient Demographics and Clinical Characteristics for Qualitative Synthesis.

128 For qualitative synthesis, 1845 patients from 191 studies were included. The median 129 average patient age was 50 years, while the median average percentage of female patients was $13072.15 \%$ (Table 1). BBA location was provided in 184 of 191 studies and 93.75\% (1755/1872) 131 were found along the ICA, whereas $1.60 \%$ (30/1872) and 1.50\% (28/1872) were localized to the 132 middle cerebral and basilar arteries, respectively (Table 1). HH scores were reported for 97 of 133191 studies and of such, 10.75\% (128/1191), 44.67\% (532/1191), 27.54\% (328/1191), 13.60\%

134 (162/1191), and 3.44\% (41/1191) of patients presented with HH scores of I, II, III, IV, and V, 135 respectively (Table 1$)$.

137 Quantitative Synthesis Outcomes Summary. 
139 complications were identified. Perioperative rebleeding was reported in 225 cases $(20.11 \%)$ and

140118 patients $(13.53 \%)$ were retreated in the perioperative setting. Overall, short-term occlusion

141 rate and mRS values were inconsistently reported. Findings in the postoperative setting identified

142 a complete occlusion rate of $65.11 \%$ (334/513) and a follow-up (FU) mRS of 0-2 in 86.8\%

143 (729/840) of patients. Postoperative complication rates including aneurysm regrowth and

144 rebleeding were $10.56 \%$ and $5.62 \%$, respectively. Overall, the postoperative mortality rate was

$145 \quad 5.3 \%$ (69/1313). Data is summarized in Table 2.

146 Analysis of BBA Treatment Groups.

147 Open Surgery. There were 53 studies with 687 patients treated with open surgery. The 148 majority of surgical interventions involved clipping, a combination of clip and wrapping, or 149 trapping and EC-IC bypass. Of the 35 studies providing data for procedural complications, the 150 rate was $45.6 \%$ (177 of 388 patients), with the most common complications being intraoperative 151 severe vasospasm and rebleed. Perioperative mortality occurred in 57 of 592 patients (9.6\%).

152 Perioperative complication rates including retreatment, vasospasm, and rebleeding were $13.5 \%$ 153 (49 of 363), 24.6\% (80 of 325), and 30.8\% (184 of 597), respectively, as well as ischemic stroke 154 (15.2\%, 44 of 290), and hydrocephalus (38.4\%, 66 of 172). Parent artery stenosis was reported 155 in 43 of the 290 patients (14.8\%). Postoperative vasospasm and rebleed were found in $25.7 \%$ and $1568.4 \%$ of cases, respectively. Complete occlusion was found in 262 of 289 (90.7\%), while the 157 remaining 27 patients obtained either near complete or incomplete occlusion at follow-up. Of 158350 patients, $281(80.3 \%)$ were reported as achieving good mRS scores (mRS 0-2) at follow-up. 159 Postoperatively, mortality occurred in 50 of 567 (8.8\%) patients. All results provided are 160 summarized in Table 2. 
Non-FDS Endovascular. There were 42 studies with 704 patients treated endovascularly

162 without the use of flow-diverting stents. Several of the procedural techniques used included

163 stenting with or without coiling, stent-in-stent placements, and embolization. Among 29 studies,

164 there was a procedural complication rate of $14.2 \%$ (44 of 310 patients). Perioperative

165 complication rates of early retreatment, vasospasm, rebleeding were $(16.4 \%, 64 / 390),(24.6 \%$,

$16679 / 321)$, and $(9.2 \%, 38 / 414)$ as follows. Stenosis of the parent artery was found in 13 of 182

167 patients $(7.1 \%)$. The perioperative mortality rate was 5.9\% (40 of 679). Postoperatively, patient

168 mortality was documented in 19 of 627 patients (3.0\%). Postoperative rebleeding was found in

16914 of $371(3.8 \%)$ and postoperative infarction occurred in 16 of 292 (5.5\%). Complete occlusion

170 was achieved postoperatively in 219 of 373 cases (58.7\%), and at latest follow-up this number

171 increased to 82.7\%. Upon follow-up, 348 of $379(91.8 \%)$ patients achieved a good mRS score

172 (0-2), while 31 were reported to have poor mRS scores. All results provided are summarized in

173 Table 2.

174 Flow-Diversion. There were 18 studies with 125 patients treated endovascularly with

175 flow diverting stents. We identified a procedural complication rate of 9.9\% (11 of 111 patients),

176 a perioperative mortality rate of $4.8 \%$ (6 of 125), perioperative complication rates including

177 retreatment, vasospasm, rebleeding were $4.2 \%, 8.3 \%$, and $2.8 \%$ respectively. Arterial stenosis

178 was found in 7 of 69 patients $(10.1 \%)$. In the postoperative setting, patient mortality was

179 documented in 0 of 119 patients. While perioperative occlusion and $\mathrm{mRS}$ were not frequently

180 reported, in the postoperative and follow-up studies, 100 of $111(90.1 \%)$ patients maintained a

181 good mRS (0-2) and the remaining $9.9 \%$ of patients fell between mRS scores of 3 to 5 . At most

182 recent radiographic follow-up, complete angiographic occlusion was found in 98 of $110(89.1 \%)$,

183 while 12 patients obtained either near complete (3/110) or incomplete occlusion (9/110). 
184 Regrowth was found in 3 of 108 cases (2.8\%). Postoperative rebleeding was reported in 0 of 103 185 patients while postoperative infarction occurred in 2 of 79 patients (2.5\%). All results provided 186 are summarized in Table 2.

Comparative outcome variables.

Perioperative Rebleeds. A total of 1119 patients were included in this analysis. Overall effect size between the procedural techniques was 0.13 (95\% CI: 0.09-0.18) and indicated

191 statistically significant difference between the arms $(\mathrm{p}<0.001)$. Post-hoc analysis indicated that 192 patients who underwent open surgery had higher average proportion of perioperative rebleeds 193 compared to those who underwent FDS [0.26 (0.18-0.34) vs. $0.00(0.00-0.03) ; \mathrm{p}=0.011]$. Those 194 who underwent FDS had lower perioperative rebleed rates compared to those who underwent non-FDS endovascular treatment [0.00 (0.00-0.03) vs. $0.05(0.02-0.09) ; \mathrm{p}=0.049]$.

Perioperative Retreatments. A total of 872 patients were included in this analysis.

197 Overall effect size between the procedural techniques was 0.10 (95\% CI: 0.06-0.14) and

198 indicated statistically significant difference between the three techniques $(\mathrm{p}<0.001)$. Post-hoc

199 analysis indicated that patients who underwent open surgery had higher average proportion of

200 perioperative retreatment compared to those who underwent FDS [0.10 (0.04-0.16) vs. 0.01

201 (0.00-0.05), respectively; $p=0.025]$. Those who underwent FDS had lower rates of the outcome 202 compared to those who underwent non-FDS endovascular treatment [0.01 (0.00-0.05) vs. 0.16 203 (0.09-0.23), respectively; $\mathrm{p}<0.001]$. All previous results are depicted as forest plots in Figure 1.

204 Postoperative Infarction. A total of 750 patients were included in this analysis. Overall

205 effect size between the procedural techniques was 0.06 (95\% CI: 0.03-0.10) and indicated 206 statistically significant difference between the three techniques $(p=0.001)$. Post-hoc analysis 
207 indicated that patients who underwent open surgery had higher average proportion of

208 postoperative infarction compared to those who underwent FDS [0.15 (0.08-0.23) vs. 0.00 (0.00-

209 0.04), respectively; $\mathrm{p}=0.002$ ]. Those who underwent FDS had similar rates of the outcome

210 compared to those who underwent non-FDS endovascular treatment [0.00 (0.00-0.04) vs. 0.01

$211(0.00-0.03)$, respectively; $\mathrm{p}=0.682]$.

212 Complete Occlusion at Last Follow-up. A total of 723 patients were included in this

213 analysis. Overall effect size between the procedural techniques was 0.86 (95\% CI: 0.81-0.91)

214 and indicated statistically significant difference between the three techniques $(\mathrm{p}=0.034)$. Post-hoc

215 analysis indicated that patients who underwent open surgery had similar average proportion of

216 complete occlusion at last follow-up compared to those who underwent FDS [0.93 (0.86-0.98)

217 vs. 0.85 (0.75-0.94), respectively; $\mathrm{p}=0.138]$. Those who underwent FDS had similar rates of the

218 outcome compared to those who underwent non-FDS endovascular treatment $[0.85(0.75-0.94)$

219 vs. $0.79(0.70-0.87)$, respectively; $p=0.385]$. All previous results are depicted as forest plots in $220 \quad$ Figure 2.

222 Risk of Bias of Studies and Results.

223 According to the GRADE assessment, outcomes ranged from very low to moderate

224 certainty on our evidence. Some outcomes contained variable 95\% CIs, resulting in imprecision.

225 Other studies may have inconsistent number of studies for each group, resulting in inconsistency

226 for those outcomes. GRADE assessment is shown in Supplemental Table 1. According to the

227 modified NOS, scores for studies ranged from 4 to 6 . The two categories that were non-

228 satisfactory for some of the studies were inadequate follow-up duration and outcome of interest.

229 Some studies failed to define final follow-up while others included patients who were reoperated. 
230 However, those patients were excluded in our analysis. NOS scoring is shown in Supplemental 231 Table 2.

232 Additionally, it is important to note that angiographic diagnosis of a blood blister

233 aneurysm has its challenges which may lead to inconsistencies between sites ${ }^{19,20}$. The utility of

234 conventional CTA in characterizing the morphology that defines a BBA is poor and although

235 most BBAs are detected with digital subtraction angiography and 3D rotational angiography,

236 some are unidentifiable pre-procedurally ${ }^{21-23}$. Hence, discriminating between BBAs and other

237 aneurysms may be at risk of bias across studies performing either endovascular or surgical

238 repairs. This may confound results due to the inherent limitations in operative views that exist

239 between each modality and its ability to identify an aneurysm as a blood blister with and without

240 direct visualization. Future improvements in imaging techniques will likely enhance the

241 preoperative detection and morphological classification of these lesions.

\section{Discussion}

243 Our meta-analysis combined outcome data of 102 studies to assess the safety and efficacy of

244 the various techniques used in the treatment of acutely ruptured BBAs. Specifically, we aimed to

245 compare the efficacy of open surgical techniques and other endovascular modalities to flow-

246 diversion. Our findings suggest that FDS are efficacious in treating BBAs with findings that

247 suggest lower rates of perioperative rebleeds, retreatments, hydrocephalus, and vasospasm.

248 The classical presentation of a patient with a BBA is a severe $\mathrm{SAH}$ and in our quantitative 249 synthesis most patients presented with an $\mathrm{HH}$ grade II and III at $44.3 \%$ and $29.0 \%$, respectively.

250 Unfortunately, BBA diagnoses can often be delayed at the time of presentation because CTA is

251 less sensitive in diagnosing small aneurysms or those localized near the skull base. ${ }^{24}$

252 Nevertheless, these aneurysms do necessitate early intervention as the risk of enlargement and 
253 re-rupture increases as treatment is delayed. ${ }^{24}$ In terms of morphology, these aneurysms are

254 small, thin-walled, half dome-shaped bulges with a broad neck that typically occur at non-

255 branching portions of the parent vessel. They are fragile and technically challenging to treat.

256 Currently, there is no consensus on a single recommended treatment approach and a search of the

257 literature reveals no randomized controlled trials on the topic to date. Hence, our study aims to

258 add to the growing body of literature on how flow-diverting techniques compare to other

259 described approaches for the management of these challenging lesions.

260 Our study found that BBAs treated with flow-diverting stents achieved a lower overall

261 retreatment rate when compared to open surgical and other endovascular techniques.

262 Specifically, the rates of retreatment were as follows: FDS $(4.2 \%, 5 / 119)$, open surgical $(13.5 \%$,

26349 of 363), and other endovascular (16.4\% (64/390). The difference in retreatment achieved

264 significance for FDS versus open surgery $(P=0.025)$ and FDS versus other endovascular

265 techniques $(P<0.001)$. Our data comparing flow-diversion against other endovascular techniques

266 are consistent with previously published reports. For example, Rouchaud et al. compared the

267 outcomes of patients treated with FDS to those treated with reconstructive endovascular

268 techniques such as stent-assisted coiling and also found a lower rate of retreatment among those

269 in the FDS treatment group, $(6.6 \%$ versus $27.1 \%, P=0.0002) .{ }^{25}$ Our findings were also similar

270 to the literature in comparing endovascular techniques to open surgery in that although surgery

271 achieves superior rates of obliteration, it does carry a higher risk of rupture and other

272 complications. ${ }^{26,27}$ Endovascular techniques, on the other hand, carry a lower risk of rupture with

273 comparable neurological outcomes, but our findings demonstrate higher rates of retreatment

274 among this subgroup $16.4 \%$ versus $13.5 \%{ }^{2826}$ These findings are also corroborated by a

275 systematic review by Szmuda et al. where clipping was associated with higher rates of 
276 intraoperative rupture (OR 6.5; 95\% CI 1.2 to 34.3), whereas stent-assisted coiling increased the

277 likelihood of retreatment (OR 4.1; 95\% CI 1.3 to 13.1) and incomplete occlusion (OR 2.6; 95\%

278 CI 1.0 to 6.6). ${ }^{29}$ Similar findings were observed in a study by Ricciardi et al. which found higher

279 rates of perioperative mortality in the surgical subgroup at 7.4\% compared to those treated

280 endovascularly at $2.8 \% .^{30}$ They also found a higher mean mRS (3.6 versus 2.5 ) and a

281 significantly higher complication rate among those treated surgically. ${ }^{30}$ Our study adds to the

282 current understanding by implementing a comparison of FDS to these other traditionally used

283 approaches, which has yet to be explored in the literature.

284 The subgroup treated by FDS achieved significantly lower rates of perioperative rebleeds 285 compared to those treated surgically $(2.8 \%, 3 / 108$ vs $30.8 \%, 184$ of $597 ; P<0.001)$ but did not

286 reach significance when compared to other endovascular approaches $(2.8 \%, 3 / 108$ vs $9.2 \%$

$28738 / 414 ; P=0.053)$. In a systematic review of 63 studies, Gonzalez and colleagues compared the

288 outcomes of BBAs treated surgically or endovascularly. ${ }^{26}$ They found that rebleeding occurred in

$28912.5 \%$ of cases treated with stent-assisted coiling, whereas the overall perioperative rate of

290 rebleed for those treated surgically was $30 \%{ }^{25}$ In addition, Rouchaud et al. reported a rebleed

291 rate of $8.3 \%$ in cases treated endovascularly, and that rate varied between deconstructive $(11 \%$,

$29295 \%$ CI 0.9 to 21.2$)$ and reconstructive (8\%, 95\% CI 4.6 to 11.4$)$ endovascular techniques. ${ }^{25}$

293 Rouchaud et al. had relatively few FD cases but also reported lower rates of rebleed for flow-

294 diverter reconstructive techniques $(6.5 \%, 95 \%$ CI 0 to 12.8$)$ versus non-flow-diverter

295 reconstructive approaches $(8.7 \%, 95 \%$ CI 4.6 to 12.8$)$ although this did not reach significance $(P$ $296=0.06) .^{25}$

297 Although flow-diverting stents achieved lower rates of rebleed above, it is important to 298 consider antiaggregant management which balances risks of acute hemorrhagic complications 
299 with risks of thromboembolic events. The pipeline embolization devices (PED) used in flow

300 diversion are unique in that they confer a 3-to-5-fold increase in surface area compared to

301 conventional intracranial stents. ${ }^{31}$ These devices are composed of $75 \%$ cobalt/chromium and

$30225 \%$ platinum/tungsten which acts as a nidus for platelet aggregation, and therefore necessitates

303 the use of dual antiplatelet therapy. ${ }^{31}$ Gupta and colleagues disseminated a survey to

304 neurosurgeons at academic cerebrovascular centers to assess the dual antiplatelet regimen used

305 following PED placement and 100\% of respondents reported using clopidogrel and aspirin as

306 first-line agents. ${ }^{32}$ It was consistently found across studies, that clopidogrel hypo- or non-

307 responsiveness was followed with administration of aspirin/ticagrelor or aspirin/prasugrel.

308 According to a multicenter cohort study by Adeeb et al. clopidogrel non-responders were at

309 significantly higher risk of a thromboembolic event compared to responders at $17.4 \%$ versus

$3105.6 \%$, respectively. ${ }^{33}$ And despite data that half of patients show some degree of clopidogrel

311 resistance, platelet function testing (PFT) remains controversial and is not uniformly

312 performed..$^{34,32,33}$ Overall, the antiplatelet regimens for intracranial aneurysms treated with flow

313 diversion is yet to reach standard protocols across sites but use of PFT and assessment of

314 ticagrelor efficacy, now preferred over clopidogrel by the AHA, warrant further investigation. ${ }^{35}$

315 The surgical subgroup achieved the highest rate of postoperative complete occlusion $(96.3 \%$,

316 105/109), whereas flow-diversion and other endovascular techniques obtained markedly lower

317 rates at $(32.3 \%, 10 / 31)$ and $(58.7 \%, 219 / 373)$, respectively. Poorer immediate occlusion rates are

318 expected of flow-diverting stents as there is rarely immediate complete occlusion, but the low re-

319 rupture rates imply that the flow diversion effect is largely protective against re-rupture. Because

320 FDS devices both divert flow and are scaffolds for neointimal proliferation, complete

321 radiographic occlusion is often not observed until endothelialization has occurred. The time 
322 course for radiographic obliteration following FDS is thought to be variable and dependent on

323 individual aneurysm morphology. ${ }^{36}$ Notably, at post-hospitalization imaging follow-up, rates of

324 complete occlusion for the FDS subgroup markedly increased to 89.1\% (98/110). Complete

325 occlusion remained high for the surgical group $(90.7 \%, 262 / 289)$ when compared to that of the

326 non-FD endovascular group $(82.7 \%, 268 / 324)$. In a study by Scerrati et al. they found that

327 coiling conferred a higher rate of immediate complete occlusion at $63.4 \%$ compared to flow-

328 diversion at $53.7 \%$, but a comparable occlusion rate was reported at follow up. ${ }^{37}$ In a meta-

329 analysis by Peschillo et al., nearly all patients who underwent open surgery achieved early

330 complete occlusion (96.4\%), whereas only $44.5 \%$ of patients who underwent endovascular

331 treatments reached postoperative complete occlusion. ${ }^{38}$ In a meta-analysis of 31 studies

332 examining reconstructive and deconstructive endovascular techniques, the immediate

333 postoperative occlusion rate was $40.6 \%$ and increased to $72.8 \%$ overall on follow-up. $^{25}$

334 Deconstructive techniques achieved higher rates of early complete occlusion compared to

335 reconstructive techniques $(77.3 \%$ versus $33 \%, P=.0003) .{ }^{25}$ Interestingly, within the

336 reconstructive group, the FDS subgroup achieved greater long-term complete occlusion at

337 follow-up compared to those treated with other reconstructive endovascular techniques $(90.8 \%$

338 versus $69.7 \%, P=0.005) .{ }^{25}$ These results align with the outcomes concluded in our study where

339 flow-diversion trended towards being superior to other endovascular approaches on the basis of

340 angiographic outcomes at follow-up with $89.1 \%$ versus $82.7 \%$, respectively.

341 Strengths and limitations.

342 The detailed literature search spanned several databases, and studies underwent

343 assessment by multiple reviewers. While our study searched a large volume of literature, it also

344 includes decades of clinical management which makes standardization between treatment groups 
345 particularly challenging. The inclusion of older data may introduce some confounders because

346 these patients were more likely treated with open approaches while the benefits of modern ICU

347 care were lacking. In addition, there are published data to suggest that outcomes following a

348 SAH have improved over time and are likely due to optimizing ICU care along with the

349 advancement of endovascular techniques. ${ }^{39,40}$ Inconsistent reporting of antiaggregant therapy

350 across studies compromised our ability to meta-analyze these data which represents an important

351 reporting bias since antiplatelet therapy is crucial for certain treatments. Small sample sizes for

352 the FDS group, in particular, make it difficult to draw statistically significant differences

353 between groups. However, this meta-analysis is the first to directly compare the safety and

354 efficacy of FDS to both surgical and other endovascular approaches.

\section{Conclusions.}

356 Overall, our findings summarize the data of 102 studies and therefore serves as a

357 clinically useful tool to estimate treatment effects and outcomes for BBAs. Flow-diversion is a

358 promising endovascular technique for treating blood blister aneurysms. Flow-diversion offers

359 lower rates of perioperative complications when compared to other frequently used treatment

360 techniques and demonstrates comparable morbidity, mortality and functional outcomes. Studies

361 assessing long-term outcomes following flow-diversion for the treatment of blister aneurysms

362 are needed.

\section{References}

366 1. Nakagawa F, Kobayashi S, Takemae T, Sugita K. Aneurysms protruding from the dorsal 367 wall of the internal carotid artery. J Neurosurg 1986; 65(3): 303-8. 
2. Takahashi A SJ, Fujiwara S, Mizoi K, Yoskimoto T. Surgical Treatment of Chimame (Blood Blister) like Aneurysm at C2 Portion of Internal Carotid Artery. Surgery for Cerebral Stroke 1988; 16(1): 72-7.

3. Ahn JY, Cho JH, Jung JY, Lee BH, Yoon PH. Blister-like aneurysms of the supraclinoid internal carotid artery: challenging endovascular treatment with stent-assisted coiling. J Clin Neurosci 2008; 15(9): 1058-61.

4. Baskaya MK, Ahmed AS, Ates O, Niemann D. Surgical treatment of blood blister-like aneurysms of the supraclinoid internal carotid artery with extracranial-intracranial bypass and trapping. Neurosurg Focus 2008; 24(2): E13.

5. Peitz GW, Sy CA, Grandhi R. Endovascular treatment of blister aneurysms. Neurosurg Focus 2017; 42(6): E12.

6. Kida S, Tenjin H, Tokuyama T, Saito O. The Histopathological Findings of Two Nonbranching Saccular Cerebral Aneurysms. Asian J Neurosurg 2020; 15(2): 431-3.

7. Abe M, Tabuchi K, Yokoyama H, Uchino A. Blood blisterlike aneurysms of the internal carotid artery. J Neurosurg 1998; 89(3): 419-24.

8. Ishikawa T, Nakamura N, Houkin K, Nomura M. Pathological consideration of a "blisterlike" aneurysm at the superior wall of the internal carotid artery: case report. Neurosurgery 1997; 40(2): 403-5; discussion 5-6.

9. Karnati T, Binyamin TR, Dahlin BC, Waldau B. Ruptured Fisher grade 3 blister aneurysms have a higher incidence of delayed cerebral ischemia than ruptured Fisher grade 3 saccular aneurysms. Brain Circ 2020; 6(2): 116-22.

10. Rasskazoff S, Silvaggio J, Brouwer PA, Kaufmann A, Nistor A, lancu D. Endovascular treatment of a ruptured blood blister-like aneurysm with a flow-diverting stent. Interv Neuroradiol 2010; 16(3): 255-8.

11. Linfante I, Mayich M, Sonig A, Fujimoto J, Siddiqui A, Dabus G. Flow diversion with Pipeline Embolic Device as treatment of subarachnoid hemorrhage secondary to blister aneurysms: dual-center experience and review of the literature. J Neurointerv Surg 2017; 9(1): 29-33.

12. Aydin K, Arat A, Sencer S, et al. Treatment of ruptured blood blister-like aneurysms with flow diverter SILK stents. J Neurointerv Surg 2015; 7(3): 202-9.

13. Cinar C, Oran I, Bozkaya H, Ozgiray E. Endovascular treatment of ruptured blister-like aneurysms with special reference to the flow-diverting strategy. Neuroradiology 2013; 55(4): 441-7.

14. Chalouhi N, Zanaty M, Tjoumakaris S, et al. Treatment of blister-like aneurysms with the pipeline embolization device. Neurosurgery 2014; 74(5): 527-32; discussion 32.

15. Hu YC, Chugh C, Mehta H, Stiefel MF. Early angiographic occlusion of ruptured blister aneurysms of the internal carotid artery using the Pipeline Embolization Device as a primary treatment option. J Neurointerv Surg 2014; 6(10): 740-3.

16. Moher D, Liberati A, Tetzlaff J, Altman DG, Group P. Preferred reporting items for systematic reviews and meta-analyses: the PRISMA statement. PLoS Med 2009; 6(7): e1000097. 17. Guyatt GH, Oxman AD, Vist GE, et al. GRADE: an emerging consensus on rating quality of evidence and strength of recommendations. BMJ 2008; 336(7650): 924-6.

18. Wells GA, Shea B, O'Connell D, et al. The Newcastle-Ottawa Scale (NOS) for assessing the quality of nonrandomised studies in meta-analyses. Oxford; 2000. 
412 19. Bojanowski MW, Weil AG, McLaughlin N, Chaalala C, Magro E, Fournier JY.

413 Morphological aspects of blister aneurysms and nuances for surgical treatment. J Neurosurg

$414 \quad 2015 ;$ 123(5): 1156-65.

415 20. Gaughen JR, Jr., Raghavan P, Jensen ME, Hasan D, Pfeffer AN, Evans AJ. Utility of CT

416 angiography in the identification and characterization of supraclinoid internal carotid artery

417 blister aneurysms. AJNR Am J Neuroradiol 2010; 31(4): 640-4.

418 21. Kouskouras C, Charitanti A, Giavroglou C, et al. Intracranial aneurysms: evaluation using

419 CTA and MRA. Correlation with DSA and intraoperative findings. Neuroradiology 2004; 46(10):

$420 \quad 842-50$.

421 22. van Rooij WJ, Sprengers ME, de Gast AN, Peluso JP, Sluzewski M. 3D rotational

422 angiography: the new gold standard in the detection of additional intracranial aneurysms. AJNR

423 Am J Neuroradiol 2008; 29(5): 976-9.

424 23. Jayaraman MV, Mayo-Smith WW, Tung GA, et al. Detection of intracranial aneurysms: multi-detector row CT angiography compared with DSA. Radiology 2004; 230(2): 510-8. 24. Ogawa T, Okudera T, Noguchi K, et al. Cerebral aneurysms: evaluation with threedimensional CT angiography. AJNR Am J Neuroradiol 1996; 17(3): 447-54. 25. Rouchaud A, Brinjikji W, Cloft HJ, Kallmes DF. Endovascular Treatment of Ruptured Blister-Like Aneurysms: A Systematic Review and Meta-Analysis with Focus on Deconstructive versus Reconstructive and Flow-Diverter Treatments. AJNR Am J Neuroradiol 2015; 36(12):

$431 \quad 2331-9$.

26. Gonzalez AM, Narata AP, Yilmaz $\mathrm{H}$, et al. Blood blister-like aneurysms: single center experience and systematic literature review. Eur J Radiol 2014; 83(1): 197-205.

434 27. Meling TR. What are the treatment options for blister-like aneurysms? Neurosurg Rev 435 2017; 40(4): 587-93.

436 28. Golshani K, Ferrel A, Lessne M, et al. Stent-assisted coil emboilization of ruptured intracranial aneurysms: A retrospective multicenter review. Surg Neurol Int 2012; 3: 84. 29. Szmuda T, Sloniewski P, Waszak PM, Springer J, Szmuda M. Towards a new treatment paradigm for ruptured blood blister-like aneurysms of the internal carotid artery? A rapid systematic review. J Neurointerv Surg 2016; 8(5): 488-94. aneurysms: A systematic review. Clin Neurol Neurosurg 2021; 202: 106550. 31. Morton RP, Kelly CM, Levitt MR. Endovascular Treatment of Intracranial Aneurysms. Principles of Neurological Surgery (Fourth Edition): Elsevier Health Sciences; 2018. 32. Gupta R, Moore JM, Griessenauer CJ, et al. Assessment of Dual-Antiplatelet Regimen for Pipeline Embolization Device Placement: A Survey of Major Academic Neurovascular Centers in the United States. World Neurosurg 2016; 96: 285-92.

448 33. Adeeb N, Griessenauer CJ, Foreman PM, et al. Use of Platelet Function Testing Before Pipeline Embolization Device Placement: A Multicenter Cohort Study. Stroke 2017; 48(5): 132245030.

451 34. Mallouk N, Labruyere C, Reny JL, et al. Prevalence of poor biological response to 452 clopidogrel: a systematic review. Thromb Haemost 2012; 107(3): 494-506.

453 35. Levine GN, Bates ER, Bittl JA, et al. 2016 ACC/AHA Guideline Focused Update on 454 Duration of Dual Antiplatelet Therapy in Patients With Coronary Artery Disease: A Report of the 
455 American College of Cardiology/American Heart Association Task Force on Clinical Practice 456 Guidelines. J Am Coll Cardiol 2016; 68(10): 1082-115.

457 36. Giacomini L, Piske RL, Baccin CE, Barroso M, Joaquim AF, Tedeschi H. Neurovascular 458 reconstruction with flow diverter stents for the treatment of 87 intracranial aneurysms: Clinical 459 results. Interv Neuroradiol 2015; 21(3): 292-9.

460 37. Scerrati A, Visani J, Flacco ME, et al. Endovascular Treatment of Ruptured Intracranial 461 Blister Aneurysms: A Systematic Review and Meta-analysis. AJNR Am J Neuroradiol 2021; 42(3): 462 538-45.

463 38. Peschillo S, Cannizzaro D, Caporlingua A, Missori P. A Systematic Review and Meta464 Analysis of Treatment and Outcome of Blister-Like Aneurysms. AJNR Am J Neuroradiol 2016; 465 37(5): 856-61.

466 39. Dhandapani S, Singh A, Singla N, et al. Has Outcome of Subarachnoid Hemorrhage 467 Changed With Improvements in Neurosurgical Services? Stroke 2018; 49(12): 2890-5.

468 40. Lovelock CE, Rinkel GJ, Rothwell PM. Time trends in outcome of subarachnoid 469 hemorrhage: Population-based study and systematic review. Neurology 2010; 74(19): 1494470501.

\section{Figure legends}

475

476 Figure 1. Meta-analysis shows comparison of perioperative outcomes for each treatment group.

477 The effect size (ES) for each group is represented by a diamond and provided

478 numerically with its corresponding 95\% CI. Overall P-values were calculated for each outcome.

479 When heterogeneity between groups reached significance, P-values for each treatment subgroup

480 are given with respect to FDS subgroup comparison. FDS indicates flow-diverting stents.

482 Figure 2. Meta-analysis shows comparison of postoperative outcomes for each treatment group.

483 The effect size (ES) for each group is represented by a diamond and provided

484 numerically with its corresponding 95\% CI. Overall P-values were calculated for each outcome. 
485 When heterogeneity between groups reached significance, P-values for each treatment subgroup 486 are given with respect to FDS subgroup comparison. FDS indicates flow-diverting stents.

\section{Supplemental Materials:}

489 Supplemental Figure 1 (Prisma Flow Diagram)

490 Supplemental Table 1 (Risk of bias assessment GRADE)

491 Supplemental Table 2 (Newcastle-Ottawa Scale)

492

493

494

495 
Comparison of Blister Aneurysm Treatment Techniques: A Systematic Review and Meta-

\section{Analysis}

Table 1 Qualitative synthesis patient characteristics

\begin{tabular}{ll} 
Characteristics \\
\hline \hline Age (years) \\
\hline Median average age \\
Sex $\quad$ $\quad$ \\
\hline Anedian \% female & $72.15 \%$ \\
\hline Internal carotid artery & \\
Middle cerebral artery & $93.75 \%$ \\
Basilar artery & $1.60 \%$ \\
Anterior communicating artery & $1.50 \%$ \\
Anterior cerebral artery & $1.01 \%$ \\
Posterior cerebral artery & $0.80 \%$ \\
Posterior communicating artery & $0.75 \%$ \\
Vertebral artery & $0.27 \%$ \\
Posterior inferior cerebellar artery & $0.16 \%$ \\
Hunt and Hess grade & $0.16 \%$ \\
I & \\
\hline III & $10.75 \%$ \\
IV & $44.67 \%$ \\
V & $27.54 \%$ \\
\end{tabular}


Table 2 Chief morbidity and mortality outcomes sub-divided by treatment group

\begin{tabular}{|c|c|c|c|c|}
\hline \multicolumn{5}{|c|}{ Number of events / patients } \\
\hline Outcome & Overall (\%) & Open (\%) & FDS (\%) & Endo (\%) \\
\hline $\begin{array}{l}\text { Perioperative } \\
\text { Rebleed }\end{array}$ & $225 / 1119(20.1)$ & $184 / 597(30.8)$ & $3 / 108(2.7)$ & $38 / 414(9.2)$ \\
\hline $\begin{array}{l}\text { Perioperative } \\
\text { Retreatment } \\
\end{array}$ & $118 / 872(13.5)$ & 49/363 (13.5) & $5 / 119(4.2)$ & $64 / 390(16.4)$ \\
\hline $\begin{array}{l}\text { Perioperative } \\
\text { Mortality }\end{array}$ & $103 / 1396(7.4)$ & $57 / 592(9.6)$ & $6 / 125(4.8)$ & $40 / 679(5.9)$ \\
\hline $\begin{array}{l}\text { Postoperative } \\
\text { Rebleed }\end{array}$ & $52 / 926(5.6)$ & $38 / 452(8.4)$ & $0 / 103(0.0)$ & 14/371 (3.8) \\
\hline $\begin{array}{l}\text { Postoperative } \\
\text { Regrowth }\end{array}$ & $112 / 1061(10.6)$ & $20 / 290(6.9)$ & $3 / 108(2.8)$ & $89 / 663(13.4)$ \\
\hline $\begin{array}{l}\text { Postoperative } \\
\text { Mortality }\end{array}$ & $69 / 1313(5.3)$ & $50 / 567(8.8)$ & $0 / 119(0.0)$ & $19 / 627(3.0)$ \\
\hline $\begin{array}{l}\text { Postoperative } \\
\text { Complete Occlusion }\end{array}$ & $334 / 513(65.1)$ & $105 / 109(96.3)$ & $10 / 31(32.3)$ & $219 / 373(58.7)$ \\
\hline $\begin{array}{l}\text { FU Complete } \\
\text { Occlusion }\end{array}$ & $628 / 723(86.9)$ & 262/289 (90.7) & $98 / 110(89.1)$ & 268/324 (82.7) \\
\hline
\end{tabular}


Table 3 Additional morbidity outcomes sub-divided by treatment group

\begin{tabular}{lcccc}
\hline \multicolumn{4}{c}{ Number of events / patients } \\
\hline \hline Outcome & Overall (\%) & Open (\%) & FDS (\%) & Endo (\%) \\
\hline \hline $\begin{array}{l}\text { Perioperative } \\
\text { Ischemic Stroke }\end{array}$ & $89 / 983(9.1)$ & $44 / 290(15.2)$ & $5 / 116(4.3)$ & $40 / 577(6.9)$ \\
\hline $\begin{array}{l}\text { Perioperative } \\
\text { Hydrocephalus }\end{array}$ & $103 / 370(27.8)$ & $66 / 172(38.4)$ & $19 / 97(19.6)$ & $18 / 101(17.8)$ \\
\hline $\begin{array}{l}\text { Perioperative } \\
\text { Vasospasm }\end{array}$ & $163 / 694(23.5)$ & $80 / 325(24.6)$ & $4 / 48(8.3)$ & $79 / 321(24.6)$ \\
\hline $\begin{array}{l}\text { Stenosis of Parent } \\
\text { Artery }\end{array}$ & $63 / 541(11.6)$ & $43 / 290(14.8)$ & $7 / 69(10.1)$ & $13 / 182(7.1)$ \\
\hline $\begin{array}{l}\text { Postoperative } \\
\text { Vasospasm }\end{array}$ & $89 / 447(19.9)$ & $78 / 303(25.7)$ & $1 / 33(3.0)$ & $10 / 111(9.0)$ \\
\hline $\begin{array}{l}\text { Postoperative } \\
\text { Infarction }\end{array}$ & $119 / 750(15.9)$ & $101 / 379(26.6)$ & $2 / 79(2.5)$ & $16 / 292(5.5)$ \\
\hline $\begin{array}{l}\text { Postoperative } \\
\text { Hydrocephalus }\end{array}$ & $65 / 412(15.8)$ & $56 / 239(23.4)$ & $1 / 72(1.38)$ & $8 / 101(7.9)$ \\
\hline & & & & \\
\hline $\begin{array}{l}\text { FU mRS (0-2) } \\
\text { FU mRS (3-5) }\end{array}$ & $729 / 840(86.8)$ & $281 / 350(80.3)$ & $100 / 111(90.1)$ & $348 / 379(91.8)$ \\
\hline & $111 / 840(13.2)$ & $69 / 350(19.7)$ & $11 / 111(9.9)$ & $31 / 379(8.2)$ \\
\hline
\end{tabular}




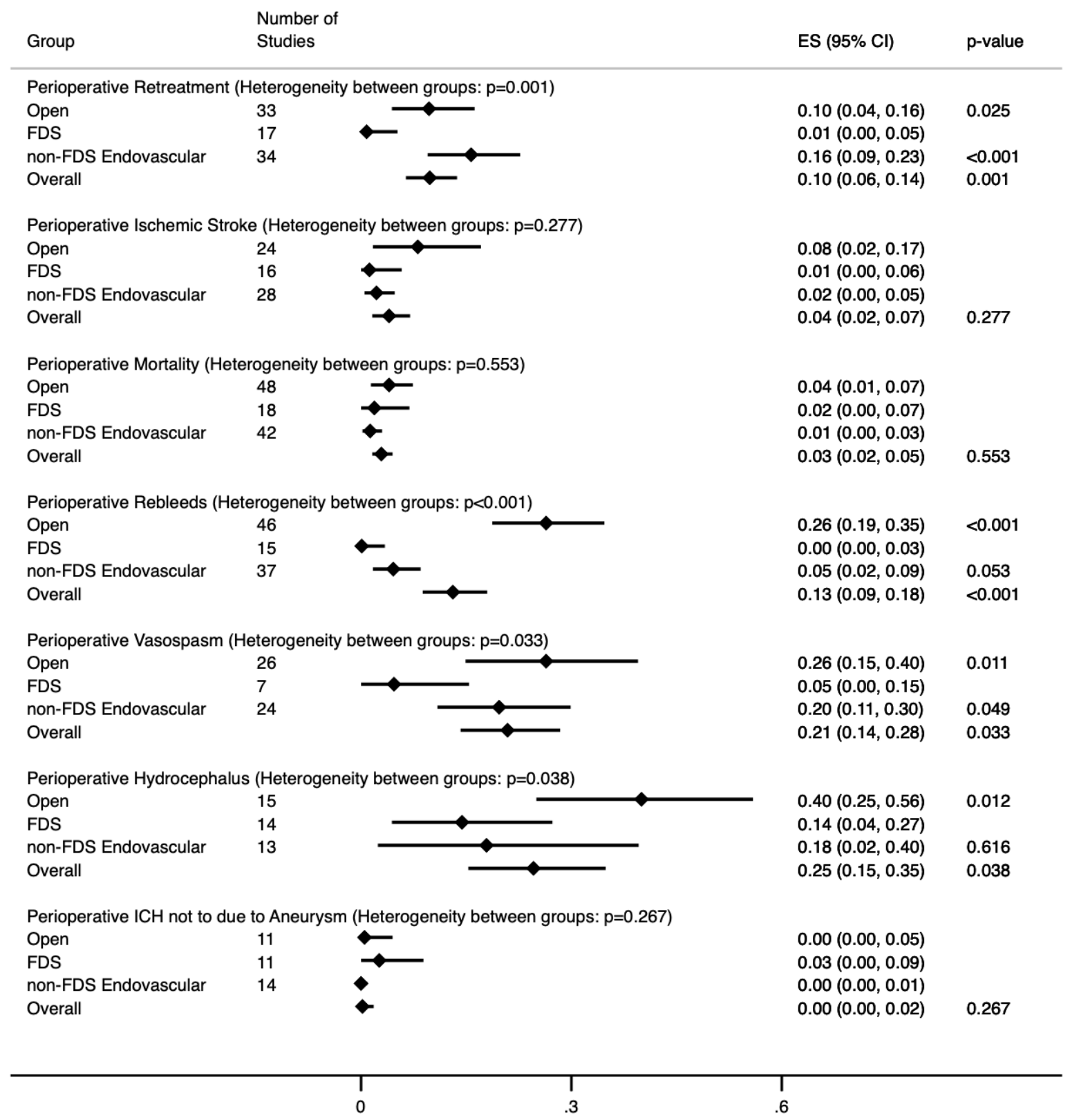

*Note: $p$-values are for comparisons to FDS unless specified for Overall 


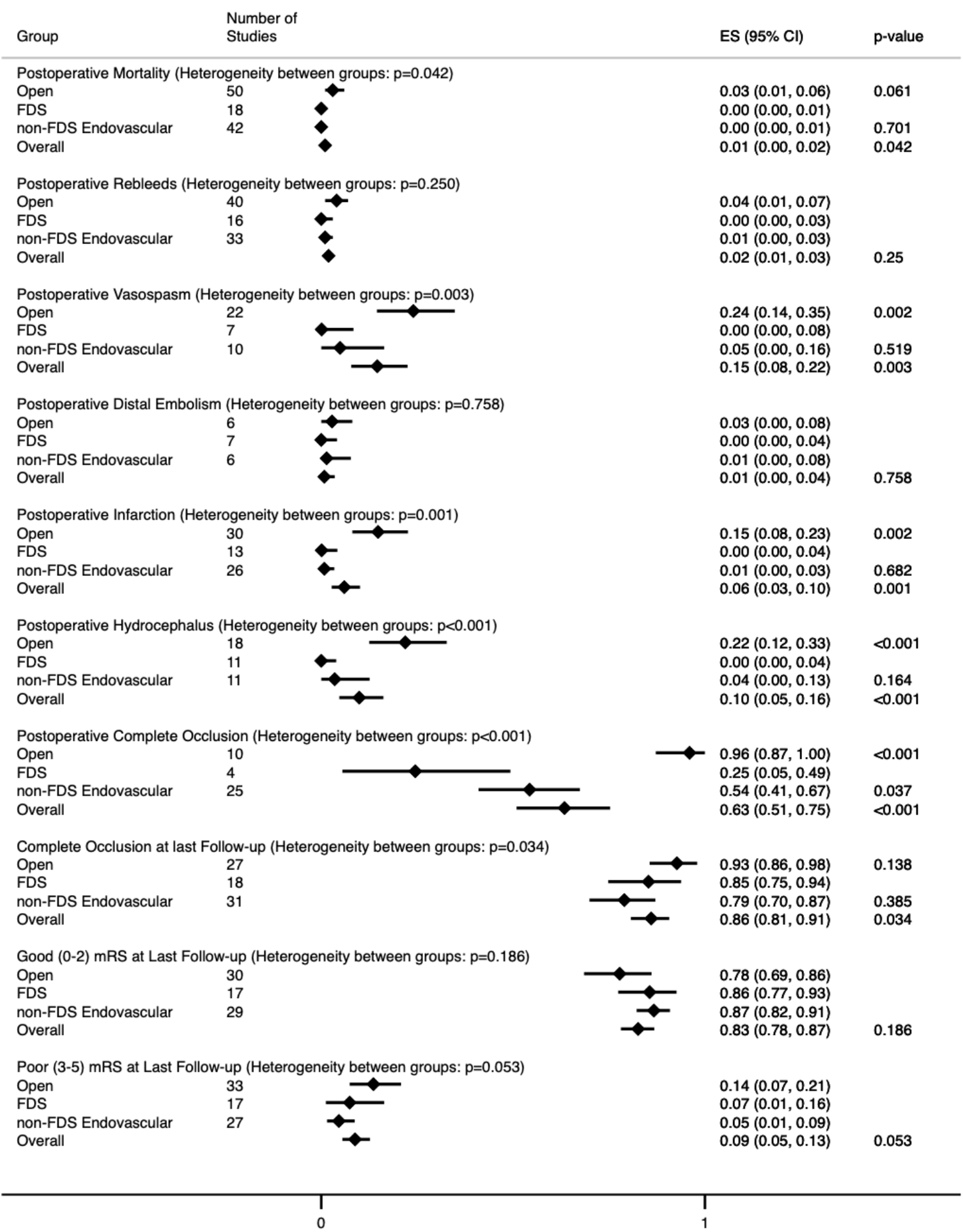

*Note: p-values are for comparisons to FDS unless specified for Overall 


\section{Contributorship Statement:}

J.N.J and A.S.H conceived and organized project. W.W. and J.N.J conceived the statistical design. A.O., A.S.H, V.E.S and S.E.R led to the literature search and data extraction and T.Y.E.A and J.N.J supervised this process with interval quality review and adjudication of any questions or disputes. W.W. performed the analysis and ASH, V.E.S and the entire authorship group reviewed and approved the statistical analysis and its interpretation via virtual meeting sessions. S.E.R, N.S.S, and V.E.S spearheaded and W.W., A.S.H and J.N.J contributed to the manuscript initial draft composition and all authors contributed to the revision process with J.N.J supervision and final approval of the final draft. 
Abbreviations used in this paper: $B B A=$ blood blister aneurysm, $A C A=$ anterior cerebral artery; $A C O A=$ anterior communicating artery; $S A H=$ subarachnoid hemorrhage; ICA = internal carotid artery; ICP = intracranial pressure; $M C A=$ middle cerebral artery; $P C o A=$ posterior communicating artery, $m R S=$ modified Rankin scale; $F D S=$ flow-diverting stents 


\section{Declaration of interests}

$\bigotimes$ The authors declare that they have no known competing financial interests or personal relationships that could have appeared to influence the work reported in this paper.

$\square$ The authors declare the following financial interests/personal relationships which may be considered as potential competing interests: 